\title{
Regiospecific conversion of substituted cinnamic acids to cinnamyl alcohols
}

\author{
Alok Kumar Mitra*, Aparna De and Nilay Karchaudhuri \\ Department of Chemistry, University of Calcutta, 92, Acharya Prafulla Chandra Road, Kolkata-700 009, India \\ E-mail:akmitra@cucc.ernet.in_Fax:91-33-23519755 \\ Manuscript received I November 2002, revised 17 February 2003, accepted 6 May 2003

\begin{abstract}
A selective reduction of cinnamic acids with oxygen functionalities in aromatic ring to the corresponding alcohols with retention of double bond using $\mathrm{ClCO}_{2} \mathrm{Et}, \mathrm{Et}_{3} \mathrm{~N} / \mathrm{NaBH}_{4}$ is reported.
\end{abstract}

In continuing our study 1 on lignan synthesis we needed to prepare a variety of cinnamyl alcohols having oxygen functionalities at the aromatic ring. Several methods ${ }^{2-7}$ are available for the reduction of carboxylic acids to primary alcohols. But, out of these methods only very few reports are there for the conversion of cinnamic acid to cinnamyl alcohol. For example, $N, N$-dimethylchloromethyleniminium chloride/ $\mathrm{NaBH}_{4}{ }^{3}, \mathrm{NaBH}_{4} / \mathrm{I}_{2}{ }^{4}$, imidazolide/ $\mathrm{NaBH}_{4}{ }_{4}^{5}, \mathrm{DCC} / \mathrm{LiBH}_{4}{ }^{6}$ and BOP reagent/NaBH${ }_{4}{ }^{7}$ have been utilized to reduce only cinnamic acid to cinnamyl alcohol ${ }^{8}$. Although a large number of biologically active aromatic naturally occurring compounds ${ }^{9}$ contain oxygen functionalities at their aromatic ring, there is no report so far on the conversion of cinnamic acids having oxygenated functionalities at the aromatic ring to the corresponding cinnamyl alcohols. These alcohols may be exploited as building blocks for the synthesis of various natural products $^{10}$. To satisfy our requirement of substituted cinnamyl alcohols in lignan synthesis, we have converted a number of cinnamic acids with oxygen functionalities to the corresponding cinnamyl alcohols using $\mathrm{ClCO}_{2} \mathrm{Et}_{2} \mathrm{Et}_{3} \mathrm{~N} / \mathrm{NaBH}_{4}{ }^{11}$ (Scheme 1). The reaction takes place regiospeci-fically on carboxylic group without affecting the double bond. To the best of our knowledge, this is the first report of the reduction of substituted cinnamic acid to cinnamyl alcohols,

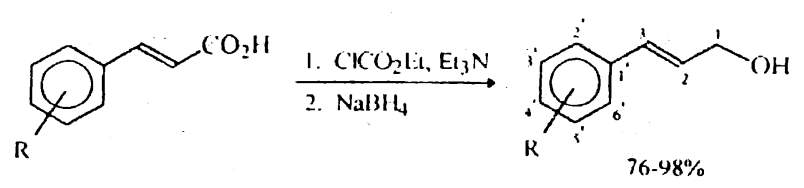

Scheme 1

which would make valuable contributions to synthetic organic chemistry. A variety of mono and disubstituted cinnamyl alcohols have been synthesized by this method in good to excellent yields (Table 1).

\section{Experimental}

The IR spectra were run on Perkin-Elmer 782 spectrophotometer. ${ }^{1} \mathrm{H}$ NMR spectra were determined in $d$-chloroform solution on Bruker AM300L instrument operating at $300 \mathrm{MHz}$ and reported in $\delta \mathrm{ppm}$ using TMS as the internal standard.

\section{General procedure :}

Cinnamic acid $(3.52 \mathrm{mmol})$ was dissolved/suspended in dry THF $(5 \mathrm{ml})$ and cooled to $-15^{\circ}$. Dry $\mathrm{Et}_{3} \mathrm{~N}^{12}(387.8$ $\mathrm{mg}, 3.84 \mathrm{mmol})$ in dry THF $(2 \mathrm{ml})$ and $\mathrm{ClCO}_{2} \mathrm{Et}^{12}(416.6$ $\mathrm{mg}, 3.84 \mathrm{mmol})$ in dry THF $(2 \mathrm{ml})$ were injected slowly and separately at a time during $2 \mathrm{~h}$ into the stirred solution at $-15^{\circ}$. The reaction mixture was warmed to $0^{\circ}$ during $1 \mathrm{~h}$ and left stirring at $0^{\circ}$ for a further period of $2 \mathrm{~h}$. The resultant precipitate was filtered off and $\mathrm{NaBH}_{4}(0.28 \mathrm{~g} .7 .4 \mathrm{mmol})$ was added to the filtrate and stirred. The reaction mixture was then quenched with $10 \%$ hydrochloric acid at $0^{\circ}$. The product was extracted wih ether $(3 \times 10 \mathrm{ml})$ and washed with brine and dried over anhydrous sodium sulphate. Evaporation of the solvent followed by the filtration on a short silica gel column afforded the pure desired products.

The IR and ${ }^{1} \mathrm{H}$ NMR spectral data of the products (entry 1-7) are given below : Entry $1: v_{\max }$ (neat) $3350(\mathrm{br}), 3020$, $2920,1650,1590,1490,960,730 \mathrm{~cm}^{-1}$; ${ }^{1} \mathrm{H}$ NMR $\delta 7.24-$ $7.08(5 \mathrm{H}, \mathrm{m}, \mathrm{Ar}-\mathrm{H}), 6.45(1 \mathrm{H}, \mathrm{d}, J 15.9 \mathrm{~Hz}, 3-\mathrm{H}), 6.20(1 \mathrm{H}$, $\mathrm{m}, 2-\mathrm{H}), 4.14\left(2 \mathrm{H}, \mathrm{dd}, J 5.6,1.2 \mathrm{~Hz}, \mathrm{CH}_{2} \mathrm{OH}\right), 3.20(1 \mathrm{H}, \mathrm{br}$ $\mathrm{s}, \mathrm{OH}) ;{ }^{13} \mathrm{C}$ NMR $\delta 134.0(\mathrm{C}-3), 128.5\left(\mathrm{C}-3^{\prime}\right), 127.7$ (C-4'), $126.5\left(\mathrm{C}-2^{\prime}\right), 123.2(\mathrm{C}-2), 64.6\left(\mathrm{CH}_{2} \mathrm{OH}\right)$ ppm; Entry 2 : $v_{\text {max }}$ (neat) 3400 (br), 3000, 1770, 1610, 1600, 1450, 1400, $1020,800 \mathrm{~cm}^{-1}$; ' $\mathrm{H}$ NMR $\delta 7.28\left(2 \mathrm{H}, \mathrm{d}, J 8.5 \mathrm{~Hz}, 2^{\prime}-\mathrm{H}, 6^{\prime}-\right.$ H), $7.03\left(2 \mathrm{H}, \mathrm{d}, J 8.5 \mathrm{~Hz}, 3^{\prime}-\mathrm{H}, 5^{\prime}-\mathrm{H}\right), 6.49$ (1H, d, J 15.9 $\mathrm{Hz}, 3-\mathrm{H}), 6.21(1 \mathrm{H}, \mathrm{m}, 2-\mathrm{H}), 4.23\left(4 \mathrm{H}, \mathrm{m}, \mathrm{CH}_{2} \mathrm{OH}\right.$, $\left.\mathrm{OCH}_{2} \mathrm{CH}_{3}\right), 3.54(1 \mathrm{H}$, br s, OH), $1.53(3 \mathrm{H}, \mathrm{t}, J 7.1 \mathrm{~Hz}$, $\mathrm{OCH}_{2} \mathrm{CH}_{3}$ ) ppm; Entry 3: $v_{\max }$ (neat) 3400-3000 (br), $2960,1770,1600,1450,1400,940,710 \mathrm{~cm}^{-1} ;{ }^{1} \mathrm{H}$ NMR $\delta$ 


Entry no. Table 1.Results of reduction of substituted cinnamic acids to cinnamyl alcohols

$7.30-7.05$ (3H, m, Ar-H), 6.98 (1H, dd, J 7.1, 1.1 Hz, 5'-H), $6.51(1 \mathrm{H}, \mathrm{d}, J 15.9 \mathrm{~Hz}, 3-\mathrm{H}), 6.27(1 \mathrm{H}, \mathrm{d}, J 15.9 \mathrm{~Hz}, 2-\mathrm{H})$, $4.23\left(4 \mathrm{H}, \mathrm{m}, \mathrm{CH}_{2} \mathrm{OH}, \mathrm{OCH}_{2} \mathrm{CH}_{3}\right), 1.30(3 \mathrm{H}, \mathrm{t}, J 7.1 \mathrm{~Hz}$, $\left.\mathrm{OCH}_{2} \mathrm{CH}_{3}\right) \mathrm{ppm}$; Entry $4: v_{\text {max }}(\mathrm{KBr}) 3400(\mathrm{br}), 3000$, $1660,1620,1450,1400,940,810 \mathrm{~cm}^{-1} ;{ }^{1} \mathrm{H}$ NMR $\delta 7.22$ $\left(2 \mathrm{H}, \mathrm{d}, J 8.6 \mathrm{~Hz}, 2^{\prime}-\mathrm{H}, 6^{\prime}-\mathrm{H}\right), 6.76\left(2 \mathrm{H}, \mathrm{d}, J 8.6 \mathrm{~Hz}, 3^{\prime}-\mathrm{H}, 5^{\prime}-\right.$ $\mathrm{H}), 6.45(1 \mathrm{H}, \mathrm{d}, J 15.8 \mathrm{~Hz}, 3-\mathrm{H}), 6.13(1 \mathrm{H}, \mathrm{m}, 2-\mathrm{H}), 4.20$ $\left(2 \mathrm{H}, \mathrm{d}, J 5.6 \mathrm{~Hz}, \mathrm{CH}_{2} \mathrm{OH}\right), 3.77\left(3 \mathrm{H}, \mathrm{s}, \mathrm{OCH}_{3}\right) \mathrm{ppm}$; Entry $5: v_{\max }$ (neat) 3400 (br), 3000,1770, 1620,1530, 1480, $1430,1050,820 \mathrm{~cm}^{-1}$; ' $\mathrm{H}$ NMR $\delta 7.26-6.52(3 \mathrm{H}, \mathrm{m}, \mathrm{Ar}-$ $\mathrm{H}), 6.57(\mathrm{lH}, \mathrm{d}, J 15.9 \mathrm{~Hz}, 3-\mathrm{H}), 6.31(1 \mathrm{H}, \mathrm{m}, 2-\mathrm{H}), 4.29$ $\left(4 \mathrm{H}, \mathrm{m}, \mathrm{CH}_{2} \mathrm{OH}, \mathrm{OCH}_{2} \mathrm{CH}_{3}\right), 3.83\left(3 \mathrm{H}, \mathrm{s}, \mathrm{OCH}_{3}\right), 2.04(1 \mathrm{H}$, $\mathrm{s}, \mathrm{OH}), 1.38\left(3 \mathrm{H}, \mathrm{t}, \mathrm{OCH}_{2} \mathrm{CH}_{3}\right) ;{ }^{13} \mathrm{C} \mathrm{NMR} \delta 153.2(\mathrm{C}=\mathrm{O})$, $151.0(\mathrm{C}-3), 139.4(\mathrm{C}-4), 135.9\left(\mathrm{C}-1^{\prime}\right), 130.0(\mathrm{C}-3), 129.0$ $\left(\mathrm{C}-6^{\prime}\right), 122.7(\mathrm{C}-2), 118.9\left(\mathrm{C}-5^{\prime}\right), 110.1\left(\mathrm{C}-2^{\prime}\right), 64.8$ $\left(\mathrm{CH}_{2} \mathrm{OH}\right), 63.2\left(\mathrm{OCH}_{2} \mathrm{CH}_{3}\right), 55.7\left(\mathrm{OCH}_{3}\right), 14.1$ $\left(\mathrm{OCH}_{2} \mathrm{CH}_{3}\right) \mathrm{ppm}$; Entry $6: v_{\max }(\mathrm{KBr}) 3400(\mathrm{br}), 2960$, $1670,1600,1510,1450,970,800 \mathrm{~cm}^{-1}$; ${ }^{1} \mathrm{H}$ NMR $\delta 6.85$ (1H, d, J $\left.1.5 \mathrm{~Hz}, 2^{\prime}-\mathrm{H}\right), 6.74\left(1 \mathrm{H}\right.$, dd, $\left.J 8.0,1.5 \mathrm{~Hz}, 6^{\prime}-\mathrm{H}\right)$, $6.67\left(1 \mathrm{H}, \mathrm{d}, J 8.0 \mathrm{~Hz}, 5^{\prime}-\mathrm{H}\right), 6.44(1 \mathrm{H}, \mathrm{d}, J 16.4 \mathrm{~Hz}, 3-\mathrm{H})$, $6.11(1 \mathrm{H}, \mathrm{m}, 2-\mathrm{H}), 5.87\left(2 \mathrm{H}, \mathrm{s}, \mathrm{OCH}_{2} \mathrm{O}\right), 4.21(2 \mathrm{H}, \mathrm{m}$, $\left.\mathrm{CH}_{2} \mathrm{OH}\right), 1.69\left(\mathrm{H}\right.$, br s, OH) ppm; Entry $7: v_{\max }(\mathrm{KBr})$ 3400 (br), 3020, 2960,1610,1590,1470,1400,980, 810 $\mathrm{cm}^{-1} ;{ }^{1} \mathrm{H}$ NMR $\delta 6.94-6.77(3 \mathrm{H}, \mathrm{m}, \mathrm{Ar}-\mathrm{H}), 6.51(1 \mathrm{H}, \mathrm{d}, J$ $15.8 \mathrm{~Hz}, 3-\mathrm{H}), 6.26(1 \mathrm{H}, \mathrm{m}, 2-\mathrm{H}), 4.29(2 \mathrm{H}, \mathrm{dd}, J 5.8,1.0$ $\left.\mathrm{Hz}, \mathrm{CH}_{2} \mathrm{OH}\right), 3.88\left(6 \mathrm{H}, \mathrm{s}, 2 \times \mathrm{OCH}_{3}\right) ;{ }^{13} \mathrm{C}$ NMR $\delta 149.3$ $\left(\mathrm{C}-3^{\prime}, \mathrm{C}^{\prime} 4^{\prime}\right), 131.1(\mathrm{C}-3), 130.1\left(\mathrm{C}-1^{\prime}\right), 126.6(\mathrm{C}-2), 119.7$ $\left(\mathrm{C}-6^{\prime}\right), 111.3\left(\mathrm{C}-2^{\prime}\right), 109.8\left(\mathrm{C}-5^{\prime}\right), 63.7\left(\mathrm{CH}_{2} \mathrm{OH}\right), 56.0$ $\left(\mathrm{OCH}_{3}\right)$ ppm.

\section{Acknowledgement}

The authors thank C.S.I.R., U.G.C., New Delhi and the University of Calcutta for financial support.

\section{References}

1. J. Mitra and A. K. Mitra, J. Chem. Soc., Perkin Trans. 1, 1992, 1285; Indian J. Chem., Sect. B, 1992, 31, 452; 1994, 33, 953.

2. R. C. Larock, "Comprehensive Organic Transformations", VCH, New York, 1989; K. Soai, S. Yokoyama and K. Mochida, Synthesis, 1987, 647; A. Giannis and K. Sandhoff, Angew. Chem. Int. Ed. Engl., 1989, 28, 218; J. Das and S. Chandrasekaran, Synth. Commun., 1990, 20, 911; Y. Suseela and M. Periasamy, Tetrahedron, 1992, 48, 371 .

3. T. Fujisawa, T. Mori and T. Sato, Chem. Lett., 1983, 835.

4. J. V. Kanath and M. Periasamy, J. Org. Chem., 1991, 56, 5964.

5. R. Sharma, G. H. Voynov, T. V. Ovaska and V. E. Marquez, Synlett, 1995, 839 . 
6. J. M. Herbert, A. T. Hewson and J. E. Peace, Synth. Commun., $1998,28,823$.

7. R. P. McGeary, Tetrahedron Lett., 1998, 39, 3319.

8. Refs. 5 and 7 reported that the same amount of saturated alcohol was also produced.

9. W. D. MacRae and G. H. N. Towers, Phytochemistry, 1984, 23, 1207.

10. S. Hanessian and R. Leger, Synlett, 1992, 402.
11. $\mathrm{ClCO}_{2} \mathrm{Et}, \mathrm{Et}_{3} \mathrm{~N} / \mathrm{NaBH}_{4}$ have been used earlier to reduce cinnamic acid having no substitution to cinnamyl alcohol (K. Ishizumi, K. Koga and S. Yamada, Chem. Pharm. Bull., 1968, 16, 492). On utilising their reaction condition, we got an appreciable amount of saturated alcohol from cinnamic acid.

12. For entries 2,3 and $5,7.68 \mathrm{mmol}^{\circ} \mathrm{Et}_{3} \mathrm{~N}$ and $\mathrm{ClCO}_{2} \mathrm{Et}$ were used and phenolic-OH group has been converted to carbonate derivative. 